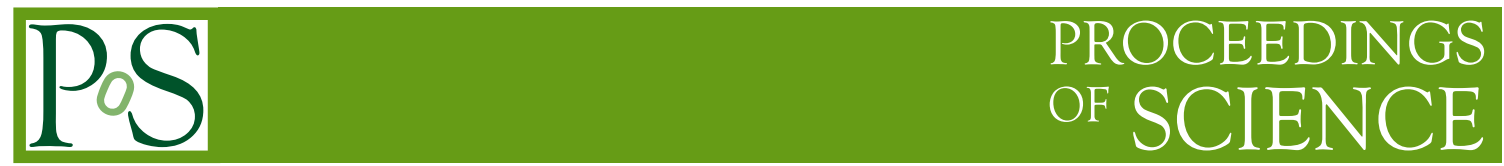

\title{
Long term radio variability of AGN
}

Talvikki Hovatta*, ${ }^{1}$ Merja Tornikoski, ${ }^{1}$ Harry J. Lehto, ${ }^{2,3}$ Elina Nieppola, ${ }^{1}$ Esko Valtaoja, ${ }^{2,3}$ Markku Lainela, ${ }^{2}$ Ilona Torniainen, ${ }^{1}$ Anne Lähteenmäki ${ }^{1}$ Margo F. Aller $^{4}$ and Hugh D. Aller ${ }^{4}$

${ }^{1}$ Metsähovi Radio Observatory, TKK, Helsinki University of Technology

Metsähovintie 114, 02540 Kylmälä, Finland

${ }^{2}$ Tuorla Observatory, University of Turku

Väisäläntie 20, 21500 Piikkiö, Finland

${ }^{3}$ Department of Physics, University of Turku

20140 University of Turku, Finland

${ }^{4}$ Department of Astronomy, University of Michigan

Ann Arbor, MI 48109, USA

E-mail: tho@kurp.hut.fi

A large number of AGN have been monitored for nearly 30 years at 22, 37 and $87 \mathrm{GHz}$ in Metsähovi Radio Observatory. These data were combined with lower frequency 4.8, 8.0 and 14.5 $\mathrm{GHz}$ data from the University of Michigan Radio Astronomy Observatory, higher frequency data at 90 and $230 \mathrm{GHz}$ from SEST, and supplementary higher frequency data from the literature to study the long-term variability of a large sample of AGN. Both the characteristics of individual flares from visual inspection and statistically-determined variability timescales as a function of frequency and optical class type were determined. Based on past behaviour, predictions of sources expected to exhibit large flares in 2008-2009 appropriate for study by GLAST and other instruments are made. The need for long-term data for properly understanding source behaviour is emphasised.

Workshop on Blazar Variability across the Electromagnetic Spectrum April 22-25, 2008

Palaiseau, France

${ }^{*}$ Speaker. 


\section{Introduction}

Active galactic nuclei (AGN) are variable across the whole electromagnetic spectrum. The radio regime is of special interest because we can directly observe the synchrotron radiation from the jet. The total flux density variations and flares seen in the flux curves are usually explained with shock-in-jet models where a disturbance creates a shock moving down in the jet, and as the shock develops we see a flare evolving from higher submm- and $\mathrm{mm}$ wavelengths towards lower radio frequencies [1,2].

We have used a sample of 90 AGN to study their long-term variability timescales $[3,4]$ and flare characteristics [5,6]. Our extensive database enables us to study the correspondence between the shock model and the observations, and also the statistical differences between the different AGN types (27 high polarisation quasars (HPQs), 33 low polarisation quasars (LPQs), 25 BL Lacertae objects (BLOs), and 5 radio galaxies (GALs)). Our sample includes bright sources that have flux density at least $1 \mathrm{Jy}$ in the active state.

\section{Variability timescales}

We used four statistical methods to study variability timescales in a sample of 80 AGN at 7 frequency bands between 4.8-230 GHz. We used the Structure Function (SF), the Discrete Correlation Function (DCF), the Lomb-Scargle periodogram, and wavelets. In the wavelet analysis we used only frequencies 22,37 and $90 \mathrm{GHz}$. In addition to analysing the timescales, we studied the properties and the differences of the methods. From the Fourier-based methods [3], the SF gives a timescale related to short variations such as the rise and the decay times of flares. The DCF and the Lomb-Scargle periodogram give the time between flares, and therefore indicates how often shocks are formed in the jet. We found that especially the Lomb-Scargle periodogram easily produces spurious spikes. The DCF is somewhat better but still suffers from treating a flux curve as one entity, a property of Fourier-based methods. Compared to Fourier-based methods, wavelets preserve the locality of the timescale i.e. show when and for how long it has been present in the flux curve. Another good property of the locality is that a change in a few points only affects the analysis locally. We lose, however, in the resolution of detecting the timescale, but when studying quasi-periodicities the accuracy is adequate. We also found that the DCF and the periodogram give very similar results as wavelets, but with wavelets it is possible to detect if the timescale is persistent or only short-lived.

As an example, in Fig. 1 the SF, the DCF and the Lomb-Scargle periodogram results at 22 $\mathrm{GHz}$ are shown for the quasar 4C 29.45. The DCF in Fig. 1b gives a timescale of 3.49 years which is very close to the timescale 3.29 years obtained with the Lomb-Scargle periodogram (Fig. 1c). Indeed, when examining the flux curve in Fig. 1a it is possible to see flares with 3 years in between them. However, the wavelet analysis of the source in Fig. 2 shows how the timescale (3.4 years from the wavelet plot) is present only in the latter half of the flux curve. Thus by using only the Fourier-based methods, one could have claimed the source to be periodic but, instead, the behaviour of the source has changed over the monitoring period. Therefore we consider it appropriate to use wavelets when quasi-periodicities in AGN are studied. We also noticed that in many sources the 

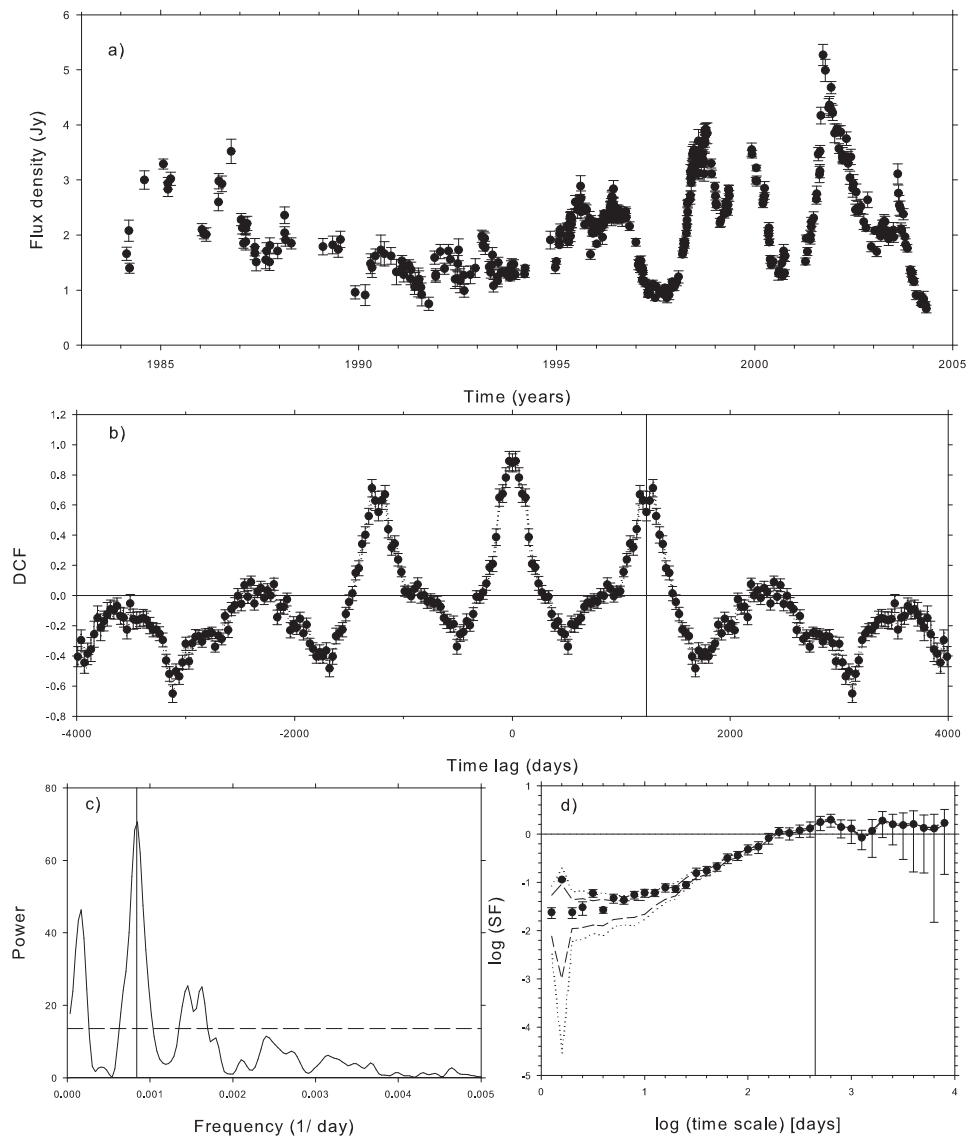

Figure 1: Analyses of the quasar 4C $29.45(1156+295)$ at $22 \mathrm{GHz}$. a) Flux density curve b) the DCF c) the Lomb-Scargle periodogram d) the SF. Timescales obtained with each method are marked by vertical lines. The periodogram timescale is 3.29 years which is 0.2 years shorter than the DCF timescale of 3.49 years. The SF gives a timescale of 1.21 years. [3]

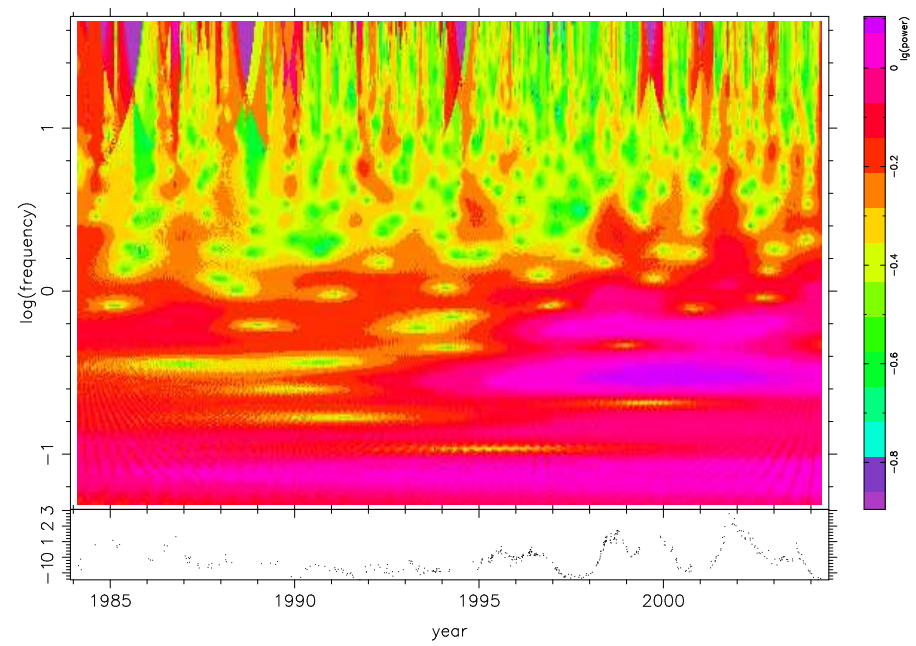

Figure 2: The wavelet analysis of $4 \mathrm{C} 29.45(1156+295)$ at $22 \mathrm{GHz}$. The timescale 3.4 is obtained from the plot at $\log \left(10^{0.53}\right)$ years. It is only present in the latter half of the flux curve starting at $\sim 1995$. [4] 


\begin{tabular}{lcccc}
\hline \hline Timescale & $14.5 \mathrm{GHz}$ & $37 \mathrm{GHz}$ & $37 \mathrm{GHz}$ z-corrected & $90 \mathrm{GHz}$ \\
\hline L-S periodogram (ave) & 9.2 & 6.3 & 4.3 & - \\
DCF (ave) & 6.7 & 4.2 & 2.6 & 3.1 \\
Wavelet (ave) & - & 4.4 & 2.8 & 2.9 \\
SF (median) & 3.2 & 1.4 & 0.7 & 1.1 \\
duration (median) & 2.8 & 1.4 & - & 2.3 \\
\hline
\end{tabular}

Table 1: Average or median timescales (in years) obtained with different methods at 3 different frequency bands. At $37 \mathrm{GHz}$ also the redshift-corrected values are shown.

timescales change slowly, get weaker in power, or disappear over long periods. Multiple timescales are common in these sources and are detected both in wavelet and in Fourier-based methods.

None of the sources in our sample showed strict periodicity in our analyses. Flares are seen in these sources on average every 4 years at 22 and $37 \mathrm{GHz}$ and the rise and the decay times of flares are between 1 to 2 years. This is also seen in Fig. 1d, where a SF timescale of 1.2 years is found for the source $4 \mathrm{C} 29.45$. The average or the median timescales at three of the frequency bands are shown in Table 1. We also studied the differences between HPQs, LPQs, and BLOs. The differences in their observed timescales were insignificant, and in all of them flares are observed, on average, in the same timescales. When we studied the intrinsic redshift-corrected timescales we found indications that BLOs have longer timescales than quasars. Quasars seem to have flares every 2 years while in BLOs they happen intrinsically every 3 to 4 years. At $37 \mathrm{GHz}$, the difference between BLOs and quasars in the DCF timescales was significant according to Kruskal-Wallis analysis, and in the wavelet timescales the BLOs differed significantly from the LPQs and there were indications that also the BLOs and the HPQs differ from each other. These results could imply that shocks are developed less frequently in BLOs than in quasars. This is contradictory to results from hydrodynamical simulations [7], which show that shocks are formed more easily in slower jet flows which are often associated with BLOs. This will be studied further in a forthcoming paper [8], where we will calculate the Lorentz factors for a large sample of sources from different classes.

Even though none of the sources in our sample are strictly periodic, many of them show episodes of quasiperiodic behaviour. Therefore we were interested to see whether we could "predict" upcoming active states in these sources. It would be useful to know which sources are in an active state when planning multiwavelength observations. In particular, we were interested in sources which would be likely to flare during the early phases of the GLAST satellite, in the time period 2008-2009. Our earlier work (e.g. [9, 10]) has shown that strong gamma-ray activity is connected to a growing flare in the radio regime, and therefore sources that are active in the radio are good candidates to be detected by GLAST.

Thus, based purely on our statistical wavelet analysis [4] combined with analyses of [3] and visual inspection of our flux curves at $37 \mathrm{GHz}$ until the end of 2007, we came up with a list of six potential sources. These sources show quasiperiodic behaviour in the wavelet analysis and in at least one Fourier-based method with such a timescale that a large flare could occur in 2008-2009. Sources exhibiting almost continuous and complex variability, such as OJ 287 and BL Lac were excluded from the study. The six sources include one GAL type object $(0007+106)$, two HPQs $(0234+285,1156+295)$, two LPQs $(0333+321,2145+067)$ and one BLO $(1749+096)$. Out of these 


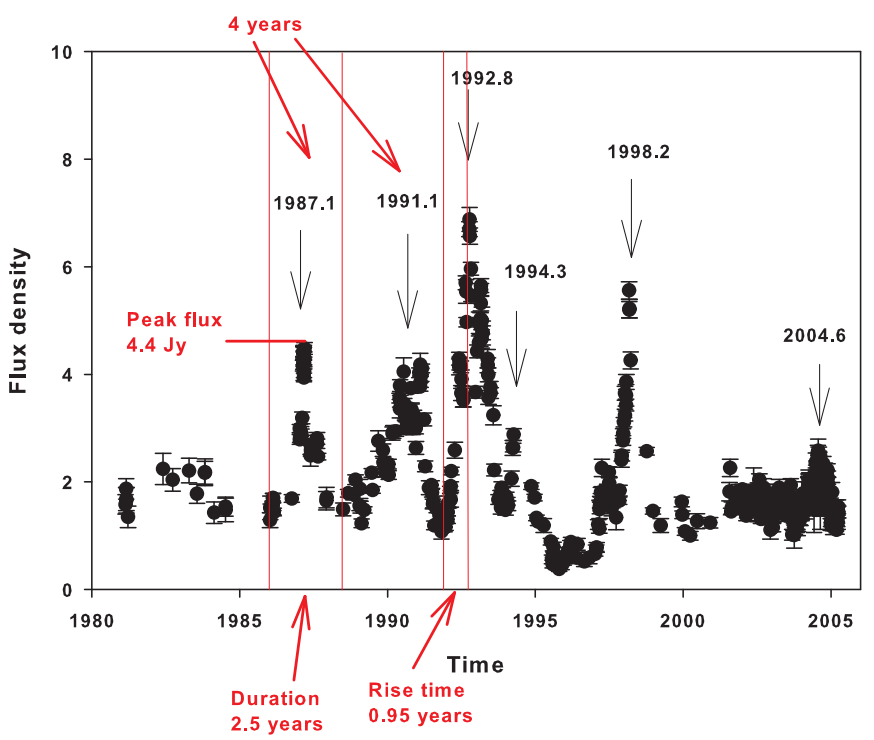

Figure 3: Flux curve of an example source $0235+164$ at $37 \mathrm{GHz}$.

six sources, two $(0007+106$ and $1749+096)$ have redshifts less than 0.5 and could be potential sources to be detected at $\mathrm{TeV}$ energies as well.

\section{Flare characteristics}

We have studied the flare characteristics of 55 sources with 159 well-monitored flares at 8 frequency bands between 4.8-230 GHz. We calculated several parameters for each flare, e.g. the amplitude, the duration, and the time delays between the frequency bands. We were not able to calculate all the parameters for all the flares at every frequency band because especially the higher frequency data were often sparsely sampled. The median peak flux density in the sample was found to be $4.5 \mathrm{Jy}$ at $37 \mathrm{GHz}$. The range of the peak flux densities in the sample was very broad, from $0.7 \mathrm{Jy}$ to $57 \mathrm{Jy}$, showing that the variability behaviour in these sources is very heterogeneous, and studies concentrating on a single source are inadequate in describing the typical behaviour of AGN. This is also seen in the flare durations which we found to range between 0.3 and 13.2 years. The median duration at $37 \mathrm{GHz}$ is 2.5 years. The long duration of flares in the radio regime also show that long-term monitoring is essential in understanding the behaviour of AGN. Short multiwavelength campaigns lasting from a couple of days to a few weeks are not sufficient in capturing a complete radio flare.

An example of an "average" blazar is in Fig. 3, where the flux curve of the BLO source $0235+164$ at $37 \mathrm{GHz}$ is shown. In the flux curve of this source we can identify the median or average values of all the parameters from the different analyses. The flare peaking at 1987 has a peak flux density of $4.4 \mathrm{Jy}$, and also the median flare duration of 2.5 years is seen in the same flare. We can also find the average time interval 4 years between the flares in 1987 and 1991 . The rise time 0.95 years of the 1993 flare also agrees with the results of the SF analysis. 
We compared the duration of the flares with the relative peak flux densities, and found that there is only a slight positive correlation of 0.15 between the values. We can see almost as high peak flux densities in flares lasting for 2 years as for flares lasting for 13 years. The correlation disappears altogether when we correct the peak luminosity and the duration for redshift and Doppler boosting (Doppler boosting factors taken from [8]). This indicates that the energy release in a flare does not increase with the duration of the flares.

Our sample includes sources from various AGN classes and are mostly blazars. The BL Lacertae objects and their timescales are studied in more detail in [6]. In addition to the flare parameters, we studied the correspondence between the observations and the shock model by Marscher \& Gear [1] and its generalisation by Valtaoja et al. [11]. We found that the observed peak fluxes and the time delays between the flares adhere quite well to the predictions of the shock model, even though there is large scatter in the data. We note that our definition of a flare is based on visual inspection and therefore many of the events classified as flares in our analysis may include multiple shocks, increasing the scatter. In our analysis we found no major differences between the quasars and the radio-bright BLOs.

\section{Conclusions}

We studied the long-term radio variability of a sample of 90 sources using statistical timescale analysis methods and visual inspection of the flare parameters. Our main results are the following:

- Fourier-based methods the DCF and the periodogram give very similar results as wavelets, but wavelets should be used when quasi-periodities are studied because they give information on the locality of the timescale. With wavelets it is possible to see if a timescale is longlasting or just a short transient phenomenon in the flux curve. DCF or periodograms can then be used to verify the timescale more accurately.

- Variability behaviour is complex and no clear periodicities could be found at radio frequencies. Episodes of quasi-periodic behaviour are common, and therefore false periodicities may be found if the temporal coverage is inadequate.

- Flares are seen, on average, every 4 years in all the source types at $37 \mathrm{GHz}$ but when intrinsic redshift-corrected timescales are studied, the quasars have shorter timescales of 2 years compared to the 3-4 years of BLOs. This could indicate that shocks are produced less frequently in BLOs than in quasars.

- Median duration of a flare is 2.5 years at 22 and $37 \mathrm{GHz}$, but the range in durations is between 0.3 and 13.2 years. When comparing the duration with intrinsic redshift- and Dopplercorrected peak luminosities, we found that the energy release in a flare does not increase with the duration of the flare.

- Flares adhere quite well to the predictions of the shock model but the scatter in the data, due to poor sampling and complicated structure of the flares, is still large. 
- By combining the median duration of flares, 2.5 years, with the average time between the flares, 4 years, we see that multifrequency campaigns should last for 5-7 years in order to catch the source in both its highest and lowest activity states.

- Long-term monitoring is essential in understanding the true behaviour of these sources at radio frequencies.

\section{References}

[1] A. P. Marscher and W. K. Gear, Models for high-frequency radio outbursts in extragalactic sources, with application to the early 1983 millimeter-to-infrared flare of 3C 273, ApJ 298 (1985) 114M

[2] P. A. Hughes, H. D. Aller and M F. Aller, Polarized Radio Outbursts in Bl-Lacertae - Part Two - the Flux and Polarization of a Piston-Driven Shock, ApJ 298 (1985) 301H

[3] T. Hovatta, M. Tornikoski, M. Lainela, H. J. Lehto, E. Valtaoja, I. Torniainen, M. F. Aller, and H. D. Aller, Statistical analyses of long-term variability of AGN at high radio frequencies, A\&A 469 (2007) $899 \mathrm{H}$

[4] T. Hovatta, H. J. Lehto and M. Tornikoski, Wavelet analysis of a large sample of AGN at high radio frequencies, $A \& A$ (2008) in press

[5] T. Hovatta, E. Nieppola, M. Tornikoski, E. Valtaoja, M. F. Aller and H. D. Aller, Long-term radio variability of AGN: flare characteristics, $A \& A 485$ (2008) $51 \mathrm{H}$

[6] E. Nieppola, T. Hovatta, M. Tornikoski, E. Valtaoja, M. F. Aller and H. D. Aller, Long-term variability of radio-bright BL Lacertae objects, (2008) in preparation

[7] P. A. Hughes, M. A. Miller and G. C. Duncan, Three-dimensional Hydrodynamic Simulations of Relativistic Extragalactic Jets, ApJ 572 (2002) 713H

[8] T. Hovatta et al. in preparation

[9] M. Tornikoski, A. Lähteenmäki, M. Lainela, and E. Valtaoja, Possible Identifications for Southern EGRET Sources, ApJ 579 (2002) 136T

[10] A. Lähteenmäki and E. Valtaoja, Testing of Inverse Compton Models for Active Galactic Nuclei with Gamma-Ray and Radio Observations, ApJ 590 (2003) 95L

[11] E. Valtaoja, H. Teräsranta, S. Urpo, N. S. Nesterov, M. Lainela and M. Valtonen, Five Years Monitoring of Extragalactic Radio Sources - Part Three - Generalized Shock Models and the Dependence of Variability on Frequency, A\&A 254 (1992) 71V 